Article

\title{
Application of UAV Photogrammetry in Displacement Measurement of the Soil Nail Walls Using Local Features and CPDA Method
}

\author{
Farid Esmaeili ${ }^{1, *}$, Hamid Ebadi ${ }^{1}$, Mohammad Saadatseresht ${ }^{2}$ and Farzin Kalantary ${ }^{3}$ (D) \\ 1 Faculty of Geodesy and Geomatics Engineering, K. N. Toosi University of Technology, \\ Tehran 1996715433, Iran; ebadi@kntu.ac.ir \\ 2 Faculty of Geomatics Eng., Tehran University, Tehran 111554563, Iran; msaadat@ut.ac.ir \\ 3 Faculty of Civil Engineering, K. N. Toosi University of Technology, Tehran 1996715433, Iran; \\ fz_kalantary@kntu.ac.ir \\ * Correspondence: farid_63@yahoo.com or faridesm@mail.kntu.ac.ir; Tel.: +98-912-342-1090
}

Received: 13 November 2018; Accepted: 6 January 2019; Published: 11 January 2019

\begin{abstract}
The high cost of land across urban areas has made the excavation a typical practice to construct multiple underground stories. Various methods have been used to restrain the excavated walls and keep them from a possible collapse, including nailing and anchorage. The excavated wall monitoring, especially during the drilling and restraining operations, is necessary for preventing the risk of such incidents as an excavated wall collapse. In the present research, an unmanned aerial vehicle (UAV) photogrammetry-based algorithm was proposed for accurate, fast and low-cost monitoring of excavated walls. Different stages of the proposed methodology included design of the UAV photogrammetry network for optimal imaging, local feature extraction from the acquired images, a special optimal matching method and finally, displacement estimation through a combined adjustment method. Results of implementations showed that, using the proposed methodology, one can achieve a precision of $\pm 7 \mathrm{~mm}$ in positioning local features on the excavated walls. Moreover, the wall displacement could be measured at an accuracy of $\pm 1 \mathrm{~cm}$. Having high flexibility, easy implementation, low cost and fast pace; the proposed methodology provides an appropriate alternative to micro-geodesic procedures and the use of instrumentations for excavated wall displacement monitoring.
\end{abstract}

Keywords: UAV photogrammetry; Close range photogrammetry; Local features; Soil Nail Wall; Measurement of deformation; Network design

\section{Introduction}

During the past two decades, with the emergence of professional digital cameras and advanced digital photogrammetric methods, the use of photogrammetric images, especially close-range photogrammetric images taken from or just above the ground surface, has become a typical practice in various applications [1]. Various needs in different contexts (e.g., quality control of industrial parts, cultural heritage, civil engineering, medicine, animation, architecture, etc.) have been addressed by such capabilities of the close-range photogrammetry as three-dimensional modelling, displacement measurement, mapping, real-time precise measurement, archiving, adaptive model-based repair, virtual visualization and so forth, so as to cope with respective challenges. Integration of close-range photogrammetry into an unmanned aerial vehicle (UAV) as a highly flexible imaging platform has led to the new branch of UAV photogrammetry [2]. The use of the UAV photogrammetric images makes possible appropriate positioning at imaging stations while enhancing the geometrical accuracy of the reference photogrammetric network. Moreover, when it comes to observing large-scale structures, 
the use of UAVs makes it possible to access otherwise inaccessible positions to implement basic photogrammetric techniques.

Displacement measurement of large-scale structures is an important application of the close-range photogrammetry, where high precision on the feature is subject to appropriate network design and local feature extraction and matching on the convergent images taken from the feature. The excavated walls are among the most sensitive structures because of importance for preventing possible collapse of neighbouring structures and the resultant financial and life damages. Compared to conventional methods for excavated wall displacement measurement (e.g., micro-geodesic methods or the use of instrumentations), photogrammetric displacement measurement offers numerous advantages. Among other advantages of the photogrammetry in this field, one may refer to their high rate of data acquisition, potential for achieving high precisions, lower observation and equipment costs and visualization nature of the observations. Application of target-based photogrammetry in such projects incurs particular limitations, including the necessity of installing and protecting the targets and restricted number of acquisition points. Should the use of artificial targets rendered infeasible, in order to use a point across a feature as the reference point for displacement measurement, one should extract their corresponding position on the images precisely. Moreover, application of UAV for geometric-spatial improvement of the imaging network in such projects can be of much help. Given that most excavation projects are performed within urban areas due to the high cost of land across such areas, the spatial limitation imposed by the presence of neighbouring constructions affects the success of reference photogrammetric imaging from the ground surface. Under such condition, application of available space by UAVs to reach suitable positions for imaging is of paramount importance in making such projects feasible.

Numerous research works have been performed on displacement measurement of large-scale structures using reference photogrammetric techniques. In 2013, Esmaeili et al. used close-range target-based photogrammetry for excavated wall displacement measurement. They showed that, using the proposed combined photogrammetric displacement adjustment (CPDA), one could achieve a displacement measurement precision of $8 \mathrm{~mm}$ provided photogrammetry targets were installed on the structure [3]. In 2015, Li et al. measured unsaturated soil deformations during triaxial testing using a photogrammetry-based method [4]. In a research published in 2010, Xiao et al. undertook transmission tower displacement measurement under various loading configurations via close-range photogrammetry. In this research, a simple innovative method based on the search for the closest neighbour after the coordinate conformation was employed to determine the corresponding targets that represented displacement at a point [5]. In 2010, Jiang et al. investigated deformations along a steel-made transportation bridge under the effect of loading using close-range photogrammetry. They proposed the refined distance constraint (RDC) method for controlling the close-range photogrammetric network and employed two-sided targets for this purpose [6,7]. Scaioni et al. (2016) measured surface changes in a down-scaled landslide model using high-speed stereo image sequences [8]. In 2010, Ozbek et al. used an instantaneous photogrammetry system to measure vibration frequency of a rotating wind turbine blade [9]. Scaioni et al. (2010) presented the development and the results of a fast method for displacement measurement based on digital images, which allowed a deformation analysis along the cross-sections of a tunnel via vision metrology [10-12]. In 2005, Fraser et al. measured surface changes of the Hobart Radio Telescope in response to a change in the angle of antenna under three scenarios, with reference to the existing vector model of the telescope surface, via close-range photogrammetry. Introducing the concept of hyper redundancy, they succeeded to achieve predetermined accuracies in their measurements [13]. Later in 2014, Cerminaro used close-range photogrammetry to improve proactive assessment of retaining walls along transportation corridors. They used continuous surface measurement and surface correspondence matching for the sake of displacement measurement [14]. In a research, Luo et al. (2017) measured the effect of pressure on large inflatable structures with the help of close-range photogrammetry. Combining the digital photogrammetry with the Delaunay triangulation, 
they could measure the pressure-induced displacements on irregular non-smooth surfaces [15]. In another work, Tsvetkov et al. (2017) used the close-range photogrammetry for deformation monitoring of load-bearing reinforced concrete [16]. For this purpose, they used IP cameras with a server to which the captured images were transferred wirelessly. In 2010, Scaioni et al. presented the development and implementation of three image-based methods for displacement detection and measurement at a vast number of points in laboratory testing on construction materials. They further compared the use of artificial targets versus dense correspondence matching without such targets for displacement measurement of a concrete beam under pressure. In both cases, it was possible to dynamically monitor the structure displacement with the sufficient accuracy [12]. With respect to displacement measurement using UAV-captured images, Nocerino et al. (2013) reported the results of an integrated $\mathrm{UAV}$ and terrestrial photogrammetric survey realized in the archaeological site of the Roman theatre in Ventimiglia, Italy. They could achieve displacement measurement accuracies as high as $4 \mathrm{~mm}$ using surface modelling based on the UAV images [17]. Naumann et al. (2014) generated digital surface models (DSM) with the help of UAV-captured images to detect deformations or shape changes occurred to dike constructions or similar structures over time [18].

In most of the mentioned research works, photogrammetric targets were used as base points for feature extraction and structural displacement measurement. Some other works were founded on surface displacement measurement. Design of the photogrammetric network has been based on the required accuracy in the specific project. Scale bars were used to indicate a reference length for the network control and exactly match the results to real scale features. In some projects (e.g., wind turbine vibration measurement [9]), real-time measurements were undertaken using multiple synchronized cameras. The sensor used in most of such projects was a non-metric RGB camera. In total, existing research works indicate that, application of photogrammetry in displacement measurement of large-scale structures is superior over similar methods in many aspects, including lower cost, faster rate of observation, high accuracy, suitable visualization power based on the acquired images, no need to touch the feature, and, if needed, the required capabilities for archiving and performing the calculations. Other methods typically used for displacement measurement of large-scale structures include laser scanners, GPS (Global Positioning System) systems, micro-geodesic techniques and instrumentation methods, to name a few. Figure 1 presents a comparison among different methods used for displacement measurement of large-scale structures, including photogrammetry, micro-geodesy, geodetic GPS systems and instrumentation. The figure is based on a review on related literature. The methods were scored based on a $0-5$ scale, with a higher score in each parameter indicating better performance of the considered in that parameter.

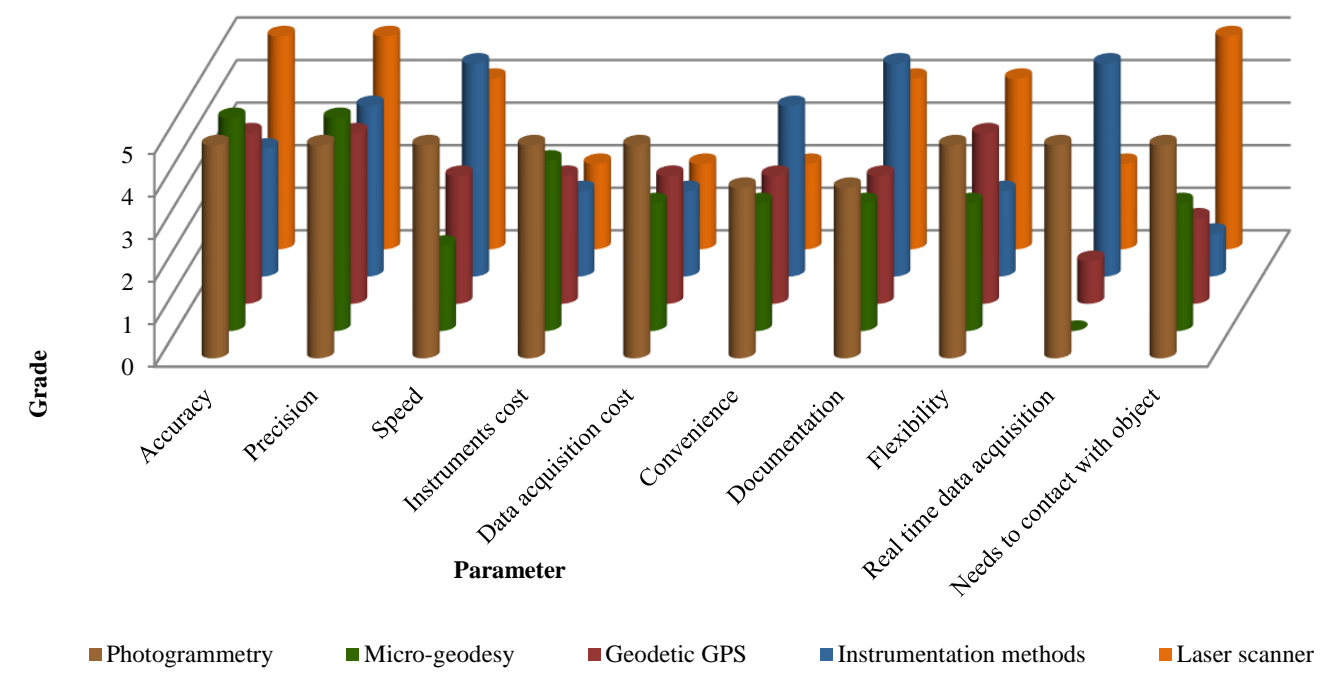

Figure 1. A qualitative comparison between close-range photogrammetric method and other methods in the measuring displacement of large-scale structures. 
According to Figure 1, when it comes to the accuracy and precision of displacement measurement, the close-range photogrammetry provides the same performance as those of micro-geodesy and laser scanners. Although the close-range photogrammetry is capable of achieving precisions in the order of microns but for most part, displacement measurement of large-scale structures requires precisions in the order of millimetres that are available through all of the three methods. When geodetic GPS systems are used for such a purpose, the results are generally less accurate than those of the micro-geodesy, as far as the height parameter is concerned. When using instrumentation, achievement of adequately precise results depends on the way the instrument is installed and the conditions under which it is maintained; in this case, it is often infeasible to undertake as precise measurement of absolute displacement along three dimensions as those achievable via micro-geodesy or photogrammetry. Considering the rate of sampling, given the nature of imaging in the close-range photogrammetry method, the rate is so high. The results of instrumentation can also be at hand immediately upon installing the required equipment. However, the need for numerous length and angle readings at various stations at each epoch in the micro-geodesy method has made this method the slowest one when it comes to the rate of observation. Moreover, the need for long-term placement of GPS receivers for acquiring observations is a relatively slow process, while acquiring observations using laser scanners at different stations is a time-consuming procedure. Considering the cost of the required equipment, the close-range photogrammetry is the most inexpensive method. The general equipment for photogrammetry include digital imaging camera, targets and scale bars, which are far less expensive than precise total stations, geodetic GPS receivers, laser scanners and expensive instrumentation equipment. Compared to other methods, close-range photogrammetry provides observations within shorter periods of time. In contrary to the instrumentation, the costs of installation and maintenance of the required equipment for photogrammetry are low. These make the cost of data acquisition lower than all of the other methods, declaring another superiority of the photogrammetry. In terms of ease of implementation and data acquisition, the close-range photogrammetry is much more convenient than the other methods. Given possible spatial restrictions faced in the project site, however, there are chances that the photogrammetry method encounters limitations in imaging or determining the fixed base points. By the way, such limitations are still there for the micro-geodesy, laser scanner and GPS-based methods. Thanks to its nature of imaging, photogrammetry provides large potentials for capturing details and generating archives of observations. The high rate and ease of data acquisition further contributes to the mentioned potentials. Also laser scanners provide large potentials for producing archives of observations given that the scanners are typically equipped with imaging cameras. Once installed, instrumentation equipment continuously transmits and stores the acquired data, which introduces a particular advantage of this method. Flexibility of the photogrammetry, particularly in achieving the target precision, is much higher than the other methods thanks to its ability to increase independent observations and hence boost redundancy of the images and also network control and on-feature point constraints. As of current, the only procedure offering the possibility of acquiring real-time three-dimensional images at ultrahigh rates, particularly for the structures experiencing instantaneous displacement, is the close-range photogrammetry. The capacity to employ a set of high-speed imaging cameras (such as those capturing at 1000 frames/second), has made the close-range photogrammetry an unrivalled approach in terms of the data acquisition rate. Among other methods, the close-range photogrammetry and laser scanners are capable of acquiring observations without physically touching the feature. The two methods are the only ones via which displacement measurement can be done by generating and comparing three-dimensional surfaces. In the photogrammetry, each target on a structure serves as an instrumentation sensor or a micro-geodesic target but rather exhibiting much better performance at much lower cost. In all of the investigated research works, the close-range photogrammetry had shown promising performance in displacement measurement and deformation measurement of large-scale structures.

Due to ever increasing value of land in urban areas, excavation activities have been significantly expanded in such areas during the recent past. Accordingly, keeping the excavated walls restrained 
firmly using different methods such as nailing is of paramount importance for protecting not only the structure under construction but also the neighbouring constructions. In the meantime, wall monitoring during the construction phase can prevent the occurrence of such incidents as excavated wall collapse. Figure 2 provides a view of the collapse of a nail-restrained excavated wall.

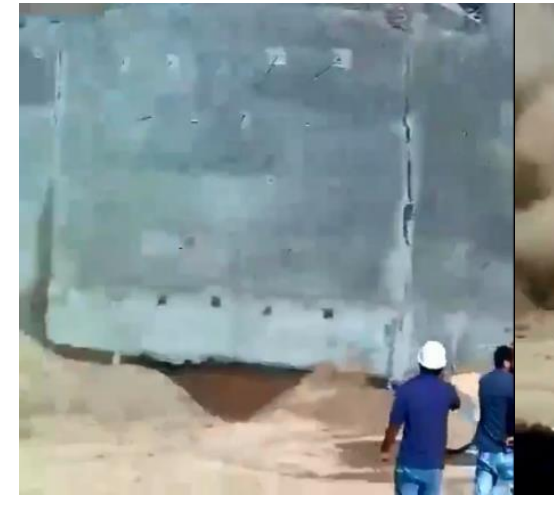

(a)

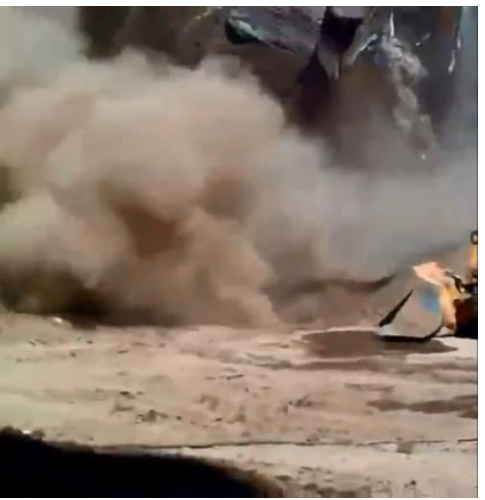

(b)

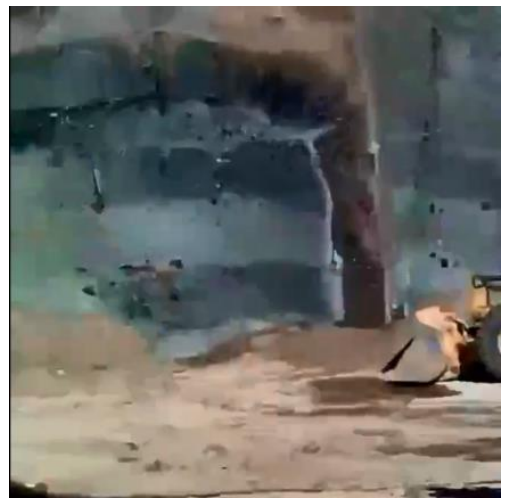

(c)

Figure 2. Collapse of a nail-restrained excavated wall in Tehran, Iran: (a) crack initiation on the wall, (b) start of the excavated wall collapse and (c) collapse of the vehicles and constructions on top of the excavated wall along with the wall into the excavated pit.

As observed in Figure 2, due to failure to monitor the wall displacement during the construction operation, the excavated wall had collapsed despite the deployment of the support structure (nailing). Micro-geodesy and instrumentation define the typical approaches to excavated wall displacement measurement. According to the assessment presented in Figure 1, high cost, low rate and difficulty of data acquisition in micro-geodesy and instrumentation methods have led to inefficient or only discontinuous monitoring in such projects. In their research, Esmaeili et al. demonstrated the potentials of photogrammetry for excavated wall displacement measurement [3]. They performed terrestrial imaging by means of photogrammetric targets as base points for displacement measurement on the feature. Application of photogrammetry-customized UAVs may provide a much efficient method, especially for urban excavation projects which suffer, for most part, from restricted working spaces. The UAVs can be stabilized at suitable-for-imaging stations beyond such working space restrictions and develop a robust imaging network to achieve high precision in the monitoring of different parts of the excavation project via photogrammetry. Moreover, using precise extraction of local features by taking metal plates at the end of nails as base points, one can monitor a wide area of the wall, thereby minimizing the need for artificial photogrammetric targets.

In the present paper, an integrated system based on UAV photogrammetry is introduced for monitoring nail-restrained excavated walls displacement. Performance of the proposed method is then evaluated on an actual project. Continuing with the work, firstly, technical structure of the system is introduced, followed by its implementation and evaluation.

\section{Materials and Methods}

In general, excavated wall displacement measurement via UAV photogrammetry can be described as shown in Figure 3. 


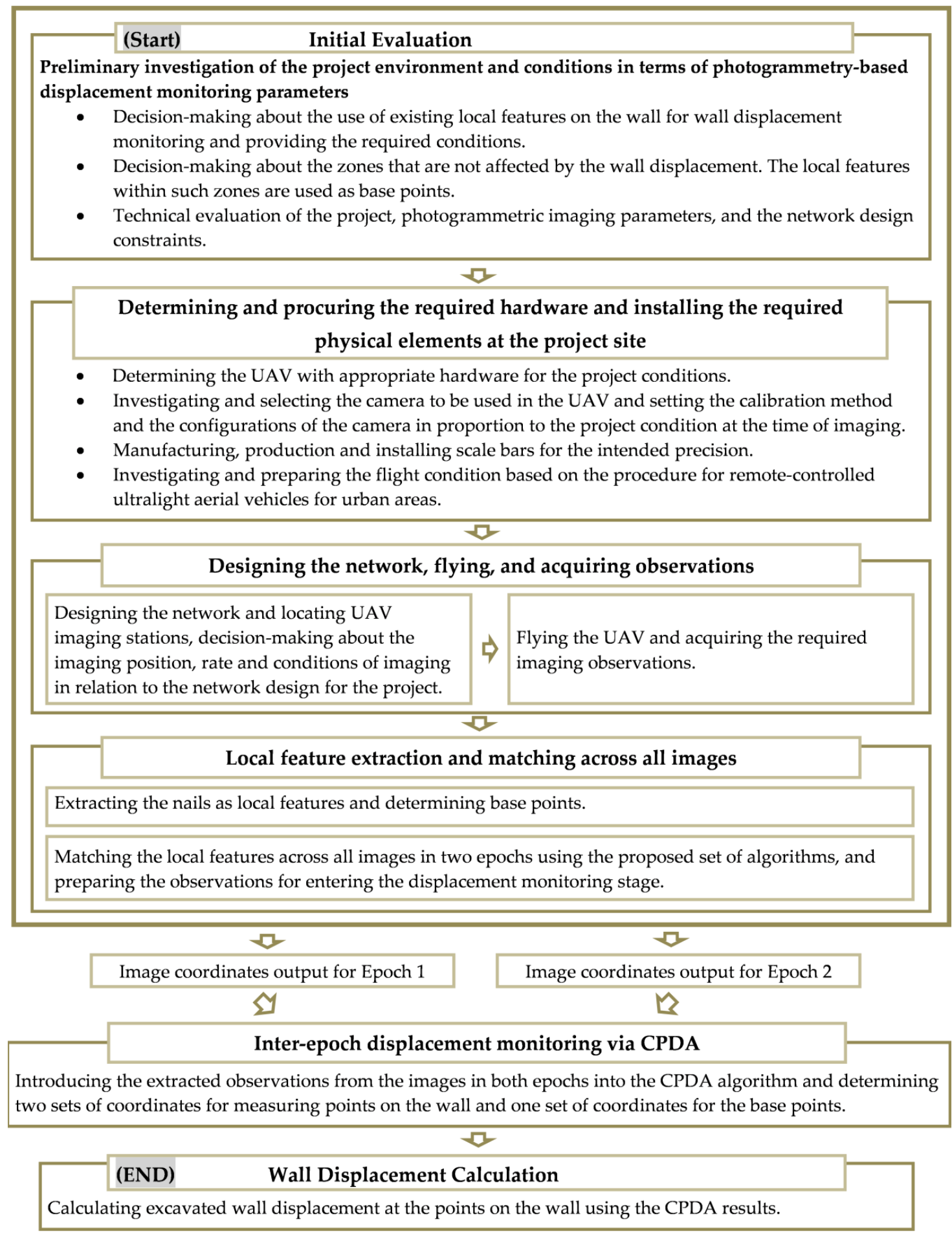

Figure 3. The flow of implementing a UAV photogrammetry system for displacement measurement of a structure.

According to Figure 3, the main steps of this process include (1) initial evaluation of the project, (2) determining and procuring the required hardware and installing and setting up the required physical elements at the project site, (3) designing the network, flying the UAV and acquiring the observations and (4) processing the acquired data to calculate final outputs in terms of wall displacement measurements. Continuing with the work, configuration of the photogrammetry method based on nailing systems is described. 


\subsection{Preliminary Investigation of the Project Environment and Conditions}

In order to implement the proposed algorithm in projects like this, a photogrammetry expert shall begin with evaluating the project in three stages:

- Firstly, one should identify a set of local features on the wall for which the local variability during the execution of the project is minimal. Given that the excavation operation is typically performed in stages, with 1-2 m of soil excavated per epoch followed by nailing and finally shotcrete spraying on the wall after each stage, the wall surface will practically experience radical changes throughout the execution of the project. In the meantime, the nails and the metal plate attached to them are not subject to such changes, so that any change in absolute position of them represents a wall displacement (Figure 4).

- Often times in nailing projects, the metal plates at the end of nails are painted in colours and numbered in rows of multiple nails. This is a typical practice for designating the excavated layers and identifying the length of the planted nails into the walls in similar projects. Given that the nails are planted into the excavated walls by some $15 \mathrm{~m}$, on average, with cement slurry injected around the nails and knowing that the metal plates are firmly screwed to the nails, principles of structural engineering imply that no systematic relative displacement is exhibited by the nails, while their absolute displacement represents that the whole wall has been displaced. On this basis, the nails naturally provide good features for the detection of the images taken via photogrammetry, where those serve as target points for determining the wall displacement. However, one should work with executive officers of the project to appropriately set the initial painting process and also take care not to spray any shotcrete on the plates. These are the local features that the algorithm proposed in this research is seeking to extract from the images (i.e., visual observations) precisely irrespective of their form, size, colour and geometry.

- In the next step, the photogrammetry expert must look for fixed positions with respect to the excavated pit according to the respective standard code [19]. Similar to the approach followed to extract the nails on the wall, appropriate fixed positions and unique local features are taken as base points for precise feature extraction from the images. This step is performed using the same algorithm for extraction and correspondence matching as that used for local features on the wall.

- In a last stage, appropriate space for imaging is determined based on the principles of photogrammetry network design [20]. To the extent possible, the presence of workshop equipment or possible auxiliary support systems such as trusses must be managed in such a way to provide suitable imaging stations with reference to the target wall while preserving the sight of imaging. Excessive wiring across the workshop space tends to negatively affect the manoeuvre power of the imaging UAV device.
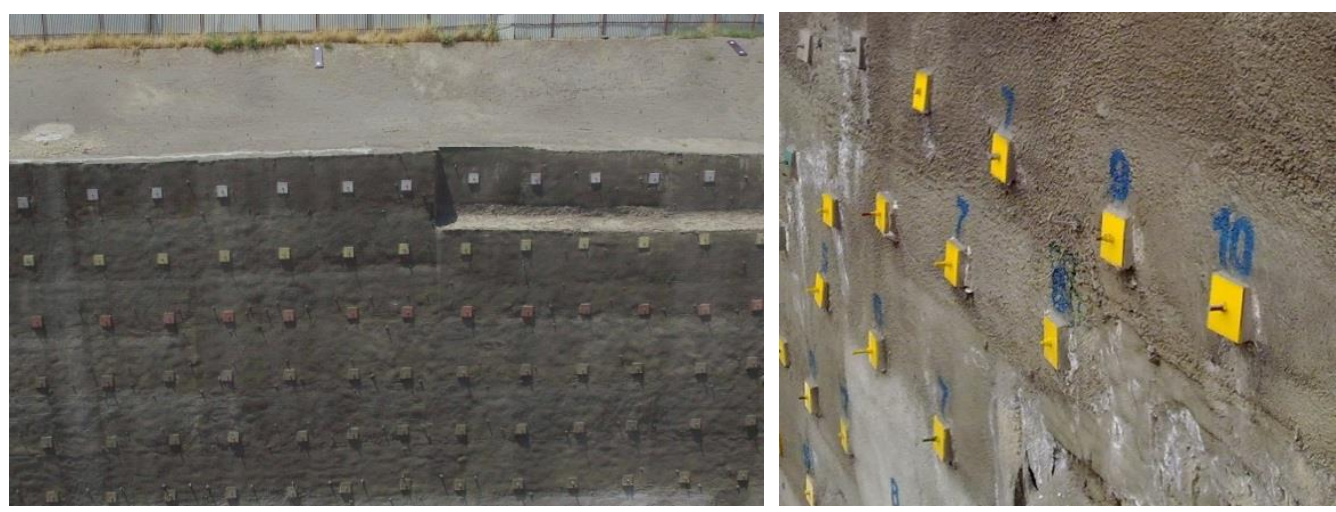

Figure 4. An example of wall nailing and the metal plate attached to the nails. 


\subsection{Determining and Procuring the Required Hardware and Installing and Setting up the Required Physical Elements at the Project Site}

In the present research, the considered structure for displacement measurement was a particular type of structure with specific properties. As such, one could configure the components of the displacement measurement system as well as the required hardware items depending on the expected properties for such projects. However, differences in the implementation conditions and the workshop environment in nailed excavations call for flexibility of the displacement measurement process to maximize its adaptability to the project. On this basis, the required pieces of equipment can be classified under two classes: "installable elements" and "used tools and equipment." These two classes are explained in the following.

If suitable conditions, in terms of the presence of appropriate nails that can be taken as local feature in the project images, are already satisfied or can be established in all parts of the project, there would be no need to install photogrammetry targets, so that the flow of displacement measurement becomes somewhat facilitated and provided with high degrees of flexibility. The metal plate attached to the nails on the wall can serve as measurement points. Using the algorithm for identifying maximally stable external regions (MSER), such regions are identified on the images and centres of mass of such regions are considered as target points for displacement measurement. However, when it comes to scaling, the scale bars have been acknowledged as the most efficient option for such a purpose. Given the possibility of precise distance measurement using the scale bars in laboratory, as compared to distance measurement using total station cameras at the project site, the scale bars can be used with appropriate distribution to address the demand for scaling across the project. Since the expected precision for the results of displacement measurement is of the order of centimetres, the sufficient tolerance of estimating the distance between the targets at the two ends of a scale bar in an excavated wall displacement measurement project is just smaller than $2 \mathrm{~mm}$. Depending on the specific set of conditions at the project site, it might be appropriate to install either fixed or portable scale bars. Regarding the need for fixed points to match the results of different epochs, if there are appropriate local features with sufficient spacing and good distribution around the excavation area for precise extraction from the images, those can be used as base points across the images. Otherwise, it is recommended to install photogrammetric targets for this purpose.

The most important piece of the used tools and equipment is the imaging camera adopted for the project. Achievement of optimal results in photogrammetry techniques is directly related to the imaging camera. Rieke-Zapp et al. performed a systematic assessment of a wide range of structures of the cameras used in photogrammetry [21]. They found that, various factors affect the final results of such projects, including the sensor structure and dimensions, the lens type and its parameters, radiometric properties, digital single-lens reflex (DSLR) structure and geometrical parameters of the camera. On the other hand, depending on the environmental conditions of excavation projects, the used of UAVs equipped with a camera provides a promising solution to achieve robust network geometry. Multi-rotor UAVs offer suitable features for excavation projects. It is obvious that the combination of the best camera on the best UAV defines the ideal for a photogrammetry project. However, considering the diversity of commercial UAVs and the fact that numerous factors affect the choice of an appropriate UAV, it is practically infeasible to acknowledge a single specific model of UAV as the best one. In this case, different criteria may differ in importance even from one user to another or from a project to another; such criteria include flight endurance, rotation angles of the UAV's gimbal, flight security sensors, dimensions and weight of the UAV, compatibility with various imaging cameras, navigation facilities and even capabilities of the flight management software. The specific model of imaging camera selected for a project imposes another constraint on the choice of the UAV. Accordingly, the choice of UAV is largely dependent on actual conditions, needs, users and available budget. 
When it comes to flying a UAV for an excavation project, since most of such projects are administered in urban areas, the procedures and regulations associated with flight in urban areas shall be closely observed and the required permission shall be obtained in advance.

\subsection{Designing the Network, Flying and Acquiring Observations}

Design of the photogrammetry network and the number and positions of the target local features for displacement measurement in the project are directly related to the achievable precision of the results. Before a UAV can provide the expert with a three-dimensional view, a dense imaging network shall be established across the project site. Extensive research has been performed on the design of close-range photogrammetry networks [20,22-26]. In the present research, the Fraser network design principles and constraints were used to determine the region within which the wall was imaged by the UAV [20].

In a flight for imaging purposes, it is so important to use a UAV equipped with position stabilization feature to capture images with no image motion and stretching and also to set the imaging region precisely. On this basis, the possibility to see the sight of UAV on a real-time basis on the controller will serve as a determinant factor. During the imaging operation, movement of individuals and equipment items across the project site shall be minimized. The specific hour(s) along the day during which the imaging operation is performed affects the performance of feature extraction from the captured images. It is better not to have long shadows in the image, particular along the margins of the nailing plates. Accordingly, the imaging operation is better to be undertaken during the hours when the wall is fully under shadow or when it is cloudy.

\subsection{Extracting and Correspondence Matching}

By definition, a local feature is a pattern of image which is uniquely distinctive of its neighbouring patterns within the part of image where it is resided [27]. Such features are typically defined based on changes in one or more image properties. For most part, such properties include changes in the grey intensity, colour and texture. Most often expressed as points, edges and closed regions across the image, these local features are used in different systems and applications, as stable points with high information content across the image. Various algorithms have been proposed for local feature extraction from an image. As the most popular ones, one may refer to the intensity-based regions [28,29], maximally stable extremal regions (MSER) [30] and segmentation-based methods [27]. Tuytelaars et al. performed a comprehensive comparison among various features of reference region extraction techniques [27]. Their results indicated that, the MSER algorithm was not only rotation-, scale and affine-invariant but also provided significantly higher levels of repeatability, accuracy, efficiency and robustness than the other typical algorithms in different applications. On this basis, the present research adopted MSER method for extracting the nailed regions on the excavated wall. The detection of MSER is related to threshold setting [27]. All stages of feature extraction, correspondence matching and displacement determination were performed by coding in MATLAB.

In this study, performance of the extraction technique was optimized by setting the thresholds on input parameters for the metal plates on top of the nails. By adjusting the range of the number of pixels falling within the MSER, aspect ratio of the extracted zones and similarity in grey intensity of the pixels within and outside region, one could optimize the MSER performance for extracting the nails on the excavated wall. Moreover, for the sake of noise attenuation, a constraint was adopted to keep near circular regions while omitting the non-circular ones. Figure 5 demonstrates an example of the regions extracted on the excavated wall using the MSER algorithm.

As can be seen in Figure 5, once finished with extracting the regions using the MSER algorithm, an ellipse was fitted to each region. Next, the centre of this ellipse was taken as a highly stable point feature for feature extraction in all images and then matching the images. 

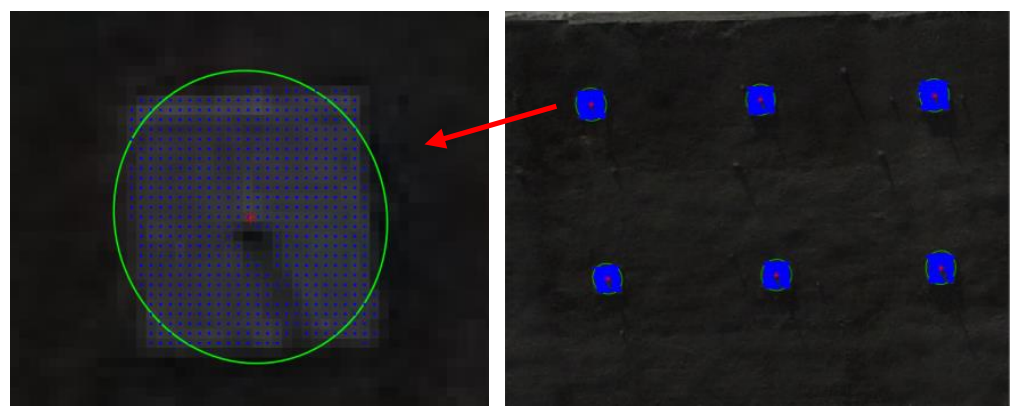

Figure 5. Metal plates on the nails, as extracted using the MSER algorithm. Further seen is the ellipse fitted to the pixels within the region and the centre of the ellipse as a highly stable point feature.

Once finished with extracting the local features, the next step is to match different points across the convergent images acquired from the wall using the UAV. Descriptors are herein determined based on weighted gradient of the image around the central point in each MSER feature. Afterwards, a feature vector was built for the central pixel of each of the MSER-extracted regions on each image. The corresponding interest points between a particular image, as reference image and the other images were identified based on similarity in the feature vector developed by the descriptors. The reference image is the one for which the incident angle of the direction of optical axis to the excavated wall is closest to $90^{\circ}$, as compared to other images. In order to ensure appropriate similarity identification, strict thresholds were considered for matching corresponding features. According to the preliminary similarities obtained in this research, coefficients of a projective geometry transformation from the reference image to the other images were determined. Accordingly, the preliminary corresponding interest points that failed to satisfy the threshold obtained upon solving the projective equations were omitted. This process of inappropriate correspondence elimination was inspired by the M-estimator sample consensus (MSAC) [31]. Figure 6 shows the initial appropriate correspondence matching between two images taken from the excavated wall by the UAV.

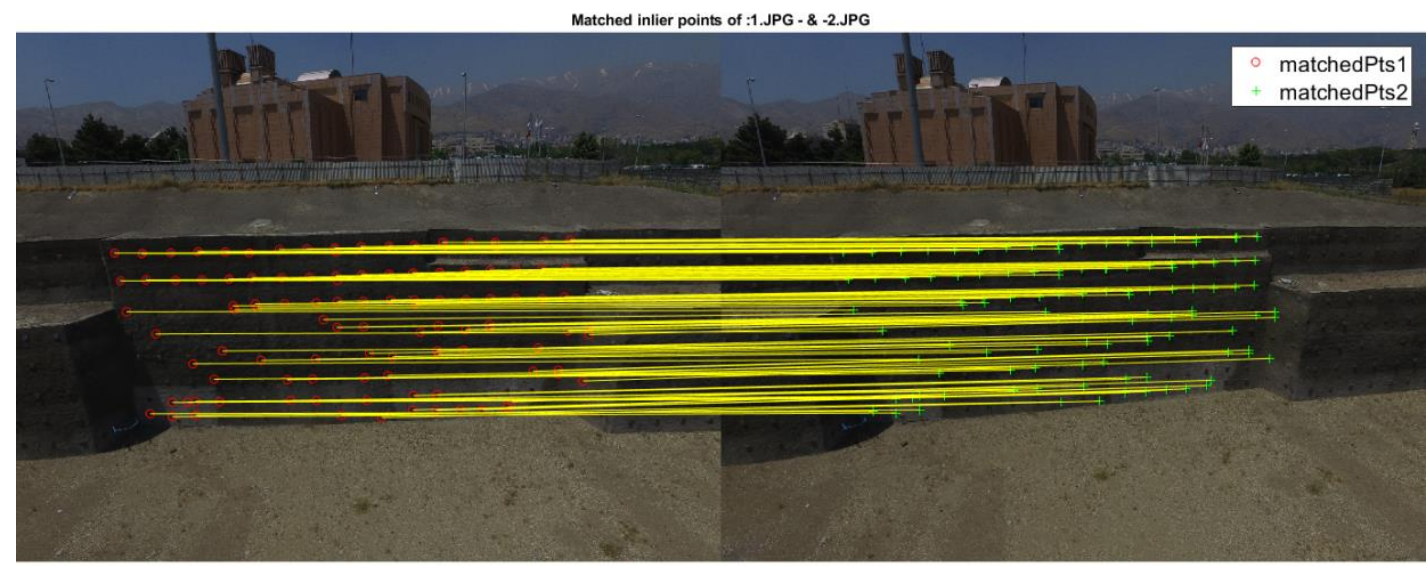

Figure 6. An example of initial correspondence matching between two images, with false correspondences omitted.

Once finished with determining the initial corresponding points, projective homography was used to project all images and points onto the reference image. The homographic images were formed according to Figure 7. 

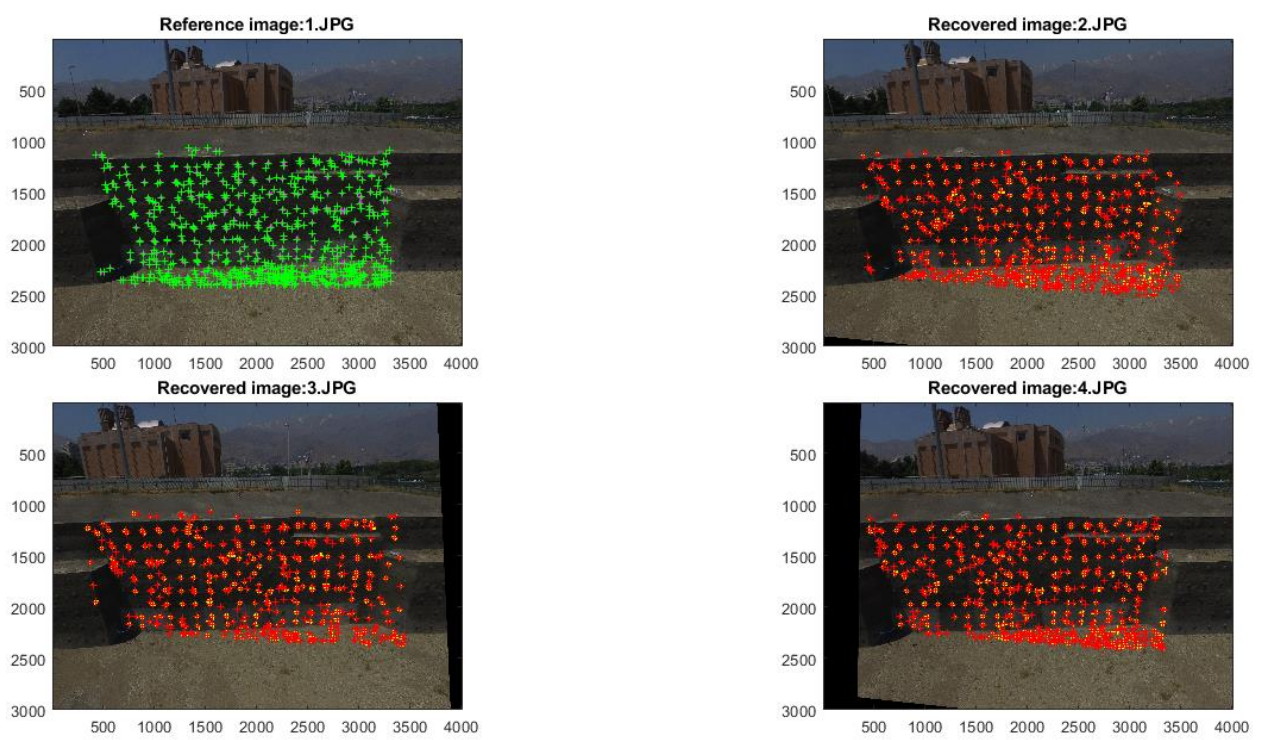

Figure 7. Three examples of the homographic images projected on the reference image.

For the corresponding points transformed into the reference image, the nearest extracted point was determined with a strict threshold. Repeated corresponding points were eliminated based on the nearest positions. On the reference image, those features for which there was corresponding features on all other images were retained, with their image and pixel coordinates determined based on the designation number of the corresponding points in all images (Figure 8).
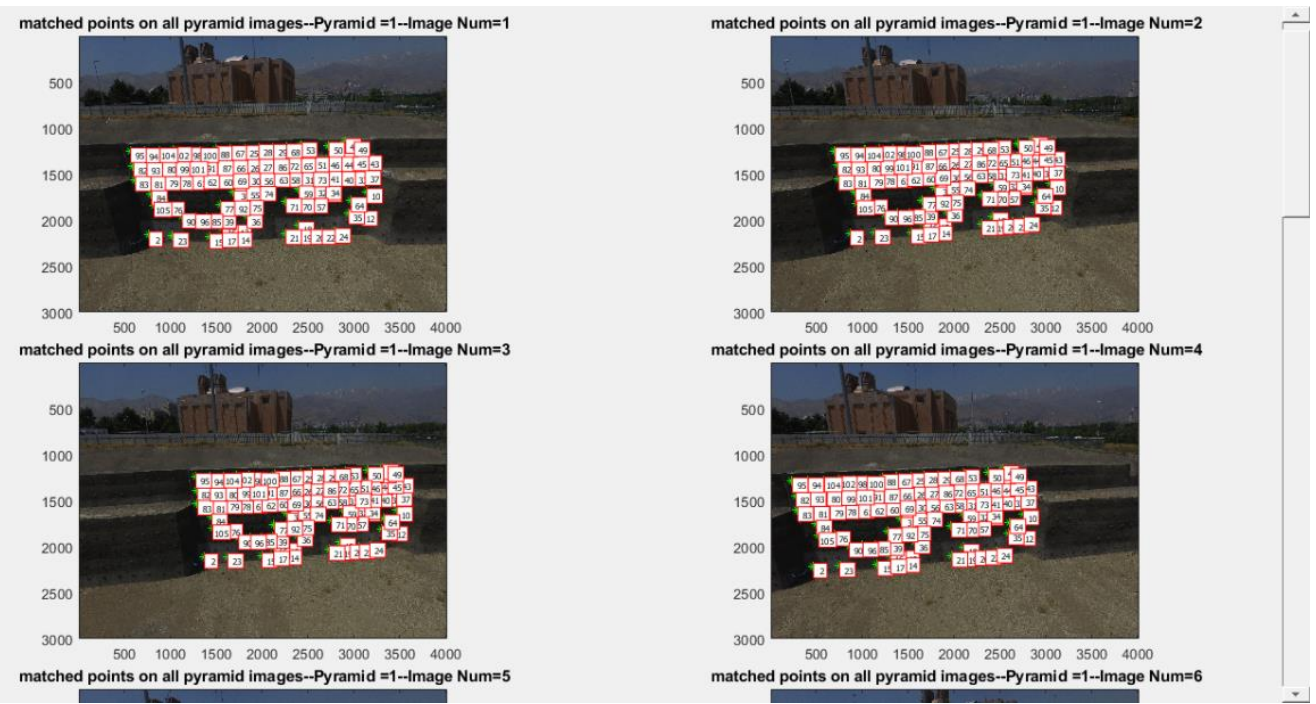

Figure 8. Final corresponding points extracted from images.

The points matching were performed across all images in the Epochs 1 and 2 . The set of corresponding points across the images in both epochs and the fixed base points were introduced into the next stage, that is, displacement measurement.

\subsection{Displacement Measurement Based on CPDA Method}

In the present research, displacement measurement was performed via combined photogrammetry displacement adjustment (CPDA) method [3]. In the CPDA, combined adjustment is applied on both epochs simultaneously by assuming common observations for base points in both epochs and independent observations at each epoch for the displaced points. In this methodology, the network 
obtained from the Epoch 1 was adjusted based on the respective internal justification, while the network derived from the Epoch 2 was simultaneously justified based on the internal justification for the second network. Coordinates of the fixed and displaced points were determined commonly between the two epochs and using images from the respective epoch, respectively. Therefore, as output, the methodology provides a set of coordinates for the fixed points along with two sets of coordinates for the displaced points, all of which reside within a common coordinate system. These two sets of coordinates for the displaced points refer to the Epochs 1 and 2. Comparing the coordinates of the displaced points, one can evaluate the displacement occurred in the two epochs. In this case, in order to scale up the dimensions to real values, scale bars are used. An important point about displacement measurement on nail-restrained excavated walls is the continuous changes in the wall surface as a result of collapse or incidence of the shotcretes originally sprayed onto the subsequent excavation layers. Therefore, except for the metal plates at the end of the nails that were originally painted and then washed regularly after each round of shotcrete spraying, no part across the wall exhibited fixed condition. This results in a situation where photogrammetry for displacement measurement cannot enjoy surface modelling techniques and hence surface comparison for wall behaviour determination is not an option in the photogrammetry-based displacement measurement methods, because most of the changes in this case are in fact resulted from changes in the shotcrete thickness in successive stages of shotcrete spraying, rather than actual wall displacement.

\section{Results}

In order to verify the propose methodology, UAV photogrammetry-based displacement measurement was implemented and evaluated in an excavation project. The case study considered in this section was stabilization of a 12-m deep excavated pit in Seoul Street (Tehran, Iran). As shown in Figure 9, the project site was located in the south of the National Committee of Olympics in Seoul Street, Tehran, Iran.

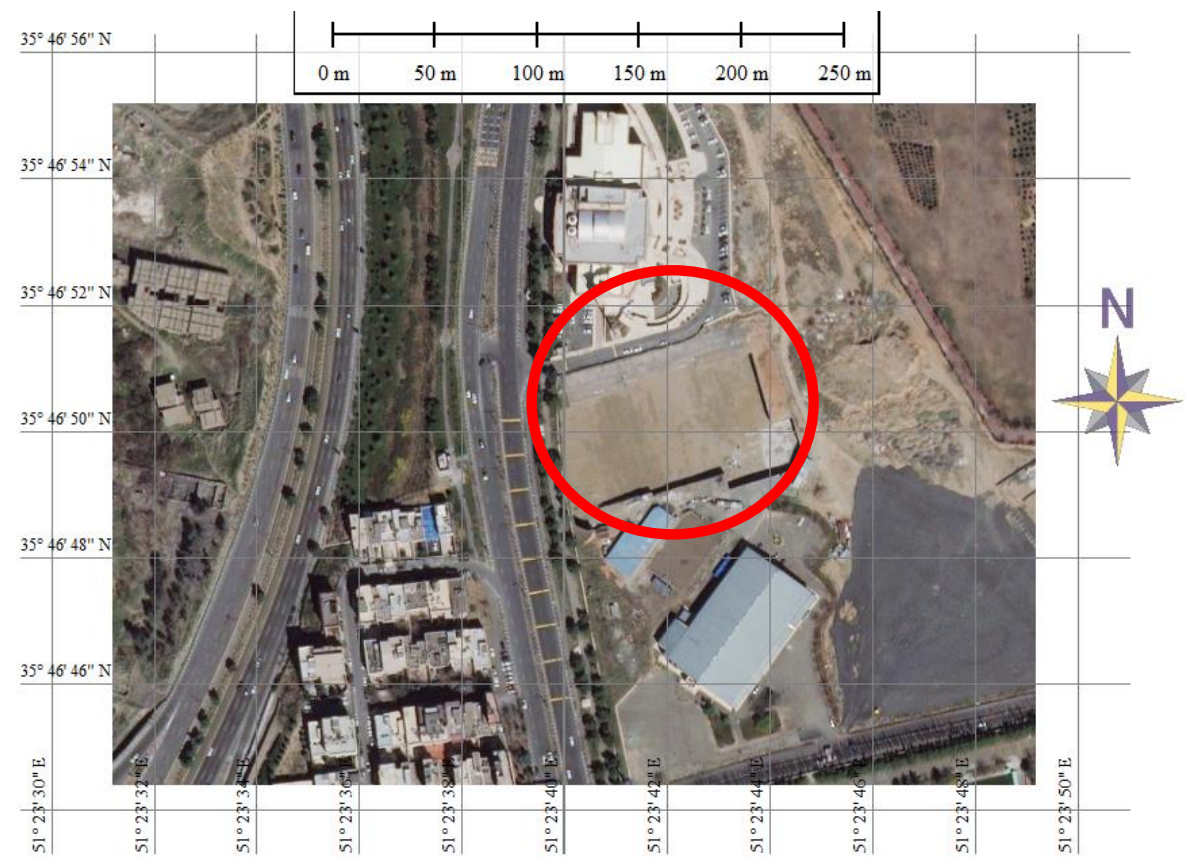

Figure 9. Introduction and location of the studied project.

\subsection{Project Conditions and Designing the Network}

The design of a UAV photogrammetry network plays a crucial role in achieving the expected precisions. In this section, we begin with explaining special conditions of such projects that affect 
the design of the photogrammetry network and then proceed to present the design of network for such projects.

The dominant soil type covering the Seoul project site was sandy soil. Ultimate excavation depth was $12 \mathrm{~m}$. In the course of imaging for displacement measurement, the excavation operation was shut down for several months. For the purpose of this project, displacement measurement was performed between two epochs spaced by some $10 \mathrm{~min}$. Given that no drilling operation was in progress and the walls were relatively stable, it was expected not to see any displacement between the observations acquired in the two epochs. Moreover, the affected horizontal distance behind the nailed wall was determined as some multiplication of the wall height and deflection angle with respect to vertical direction, as expressed in Equation (1) [19]:

$$
D_{D E F}=C \times H \times(1-\tan \alpha) \rightarrow D_{D E F}=0.8 \times 12 \times(1-\tan 0)=9.6 \mathrm{~m}
$$

where $D_{D E F}$ is the horizontal distance behind the wall that is affected by the wall displacement, $H$ and $\alpha$ are the wall height and deflection angle with respect to vertical direction, respectively and $C$ is a coefficient determined from Table 1 based on the soil type. $\left(\delta_{h} / H\right)_{i}$ is a ratio dependant on the soil conditions " $i$ " indicated in the table below [19].

Table 1. The values of $\left(\delta_{h} / H\right)_{i}$ and $C$ coefficients as functions of soil type [19].

\begin{tabular}{cccc}
\hline Variable & Weathered Rock and Stiff Soil & Sandy Soil & Fine-Grained Soil \\
\hline$\left(\delta_{h} / H\right)_{i}$ & $1 / 1000$ & $1 / 500$ & $1 / 333$ \\
$C$ & 1.25 & 0.8 & 0.7 \\
\hline
\end{tabular}

For sandy soil and excavation height of $12 \mathrm{~m}$, according to Equation (1), the horizontal distance behind the wall within which there were chances of being affected by the wall displacement was about $10 \mathrm{~m}$. Figure 10 demonstrates the excavation area and its dimensions.

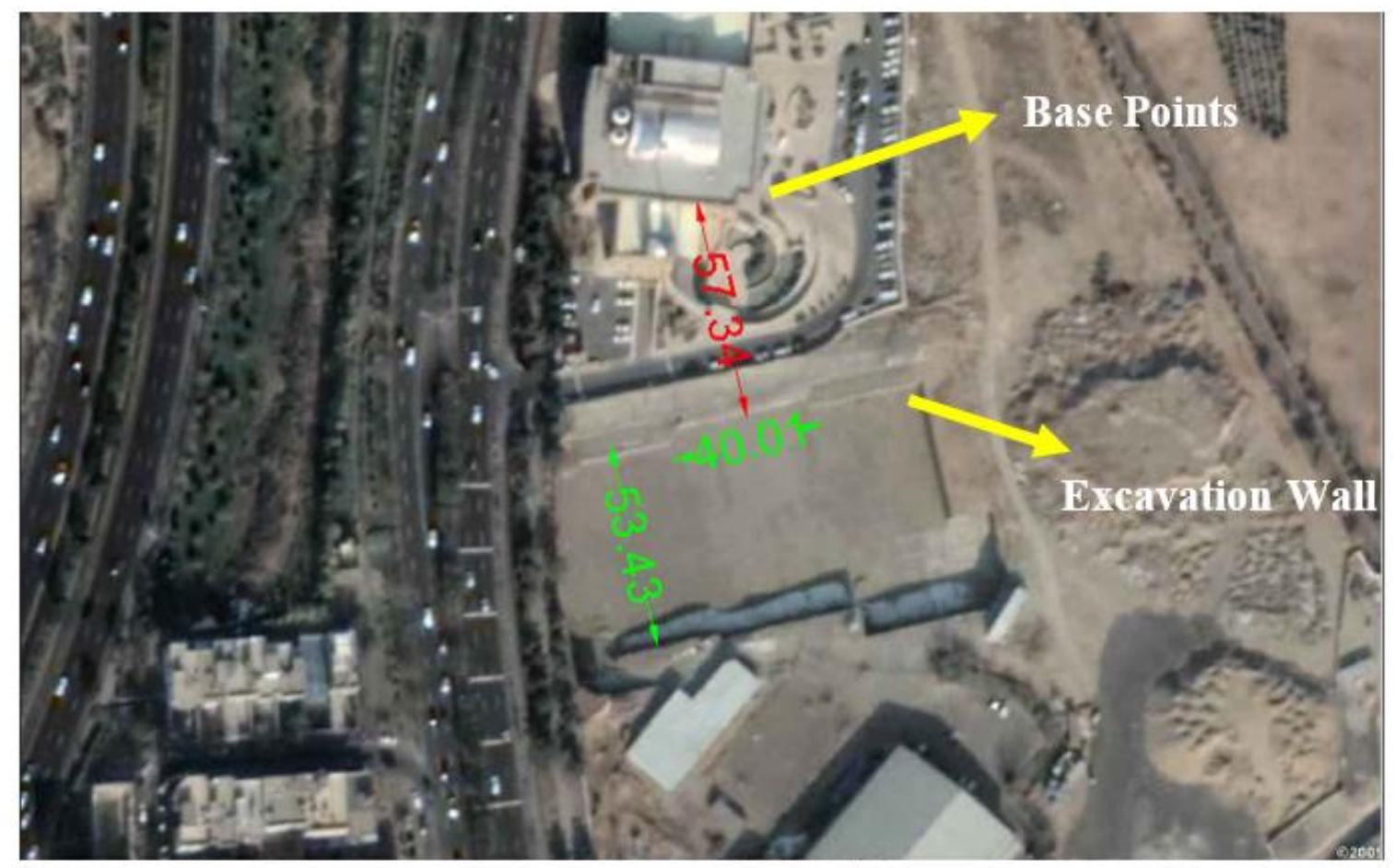

Figure 10. The excavation area and its dimensions.

According to Figure 10, the fixed points were at a distance of about $57 \mathrm{~m}$ to the excavation pit, which was beyond the 10-m distance determined in Equation (1). In both imaging epochs, focal length 
of the camera was fixed at $5 \mathrm{~mm}$. Given the average distance between the camera and the feature, this provided an imaging scale of 1:8000. The employed Sony EXMOR camera was equipped with a sensor of $4.62 \times 6.16 \mathrm{~mm}$ in dimensions. The camera captured images of 4000 pixel by 3000 pixel, which implied that each pixel on the sensor is $0.00154 \mathrm{~mm}$. Based on the image coordinate measurement resolution, the worst measurement precision was represented by 1 pixel. At each imaging station, the camera was rotated by 90 degrees to capture 2 images, with the network design factor in the worst case taken as 0.8. Under these conditions, according to Fraser's empirical equation for network design (Equation (2)), terrestrial coordinate measurement precision for the targets was calculated as follows [20,32]:

$$
\bar{\sigma}_{c}=\frac{q}{k^{1 / 2}} S \sigma \rightarrow \bar{\sigma}_{c}=\frac{0.8}{\sqrt{2}} \times 8000 \times 1 \times 1.54 \times 10^{-6}=0.0069 \mathrm{~m}
$$

where $q$ is the network design factor, $k$ is the number of images from each station, $S$ is the average imaging scale, $\sigma$ is the image coordinate measurement accuracy $(\mathrm{mm})$ and $\bar{\sigma}_{c}$ is the achievable coordinate measurement precision on the object $(\mathrm{mm})$. Therefore, even in the worst case, one could achieve a precision of about $7 \mathrm{~mm}$ in determining the coordinates of on-feature points. This was while, an increase in the number of imaging stations could justify the use of a network design factor of 0.7 rather than the 0.8 (maximum allowed). Continuing with the case study, the network design constraints are addressed.

\subsubsection{Range-Dependent Constraints}

In this research, given that the imaging operation was performed using a Phantom 3 UAV and availability of open space in front of the surveyed wall, we experienced no spatial limitation for imaging the feature. Thanks to the focal length of the camera, no problem occurred regarding the depth-of-field constraint as the minimum object-camera distance exceeded 10 times the focal length. In relation to the imaging resolution constraint, maximum allowable object-camera distance due to such resolution constraint could be calculated via Equation (3). With dimensions of about $40 \mathrm{~cm}$, central points of the steel plates at the end of nails were extracted as local features. With a focal length of 5 $\mathrm{mm}$ and CCD dimension on the sensor of $0.00154 \mathrm{~mm}$, at least 20 pixels were required to identify a plate in the image and determine its central point.

$$
D_{\text {Res }}^{\max }=\frac{D_{t} \times f \times \sin \varphi}{I_{\text {Res }} \times T_{\text {PelNo }}}=\frac{400 \times 5 \times \sin 90^{\circ}}{0.00154 \times 20^{\circ}}=65 \mathrm{~m}
$$

where $f$ is the focal length of the camera $(\mathrm{mm}), \varphi$ is incident angle of the optical direction of the camera to the nail plates, $D_{t}$ is the nail dimension $(\mathrm{mm}), T_{P e l N o}$ is the minimum number of pixels comprising the width of target local feature (normally ranging between 5 and 10 pixels) and $I_{\text {Res }}$ is the resolution of the image (pixel dimension in $\mathrm{mm}$ ). In other words, considering a resolution of 12 mega pixels (dimension of each pixel $=1.54 \mu \mathrm{m}$ ), one would encounter no problem in relation to the resolution constraint when it came to the identification of the plates of at least $40 \mathrm{~cm}$ in dimension at an average distance of $65 \mathrm{~m}$ ( 20 pixel for the width of each nail on the image). In this project, average horizontal distance of imaging stations to the wall was set to $40 \mathrm{~m}$.

Regarding the field-of-view constraint, the object-camera distance could be determined in such a way to obtain appropriate distribution of nails across each image. Equation (4) was considered to see whether this constraint is satisfied in this particular project [32].

$$
D_{F O V}^{\max }=\frac{D_{0} \times \sin (\alpha+\varphi)}{2 \sin \alpha}=\frac{40 \times \sin \left(29^{\circ}+90^{\circ}\right)}{2 \times \sin \left(29^{\circ}\right)}=54 \mathrm{~m}
$$


with $\alpha$ calculated as follows:

$$
\alpha=\tan ^{-1}\left(\frac{0.9 \times d_{0}}{2 f}\right)=\tan ^{-1}\left(\frac{0.9 \times 6.16}{2 \times 5}\right)=29^{\circ}
$$

where $D_{0}$ is the largest diameter of the object $(40 \mathrm{~m})$ and $\varphi$ is the angle between the camera's line of sight and the direction of the largest diameter of the object, which was assumed as 90 degree in the least coverage case, that is, front view. Moreover, $d_{0}$ denotes the camera sensor dimension along the length of the feature $(6.16 \mathrm{~mm})$. The focal depth was $5 \mathrm{~mm}$. As can be observed, maximum allowable object-camera distance under this constraint was $54 \mathrm{~m}$, which was satisfied with the average distance of $40 \mathrm{~m}$.

The image scale constraint expresses a threshold for the average distance between the object and different imaging stations, beyond which the point precision may no more be satisfied. For the sake of the present research, the threshold distance was calculated according to Equation (6):

$$
D_{\text {Scale }}^{\max }=\frac{D_{0} f \sqrt{k}}{q S_{p} \sigma_{i}}=\frac{40000 \times 5 \times \sqrt{1}}{0.8 \times 4000 \times 0.5 \times 1.54 \times 10^{-3}}=81168 \mathrm{~mm} \cong 81 \mathrm{~m}
$$

In this equation, $D_{0}$ is the largest diameter of the object $(40 \mathrm{~m}), f$ is focal length of the camera $(5 \mathrm{~mm}), k$ is the number of images taken at each station for each image, $q$ is the network design factor (0.8), $S_{p}$ is the expected relative precision ( 1/4000 of the feature dimension) and $\sigma_{i}$ is the image coordinate measurement error ( 0.5 pixel). As can be observed, the considered distance between the object and imaging station ( $40 \mathrm{~m}$, on average) is shorter than the maximum allowable distance obtained from Equation (6).

Respecting the number and distribution of image points constraint, practical experiences have shown that a total of 12-20 points with uniform distribution across the image provide a good pattern of data. Accordingly, since the number of points extracted in this project exceeded the 20, we had no problem regarding this constraint. Moreover, being based on reference feature extraction, the present methodology could extract adequate number of nails (as measurement points) by setting the thresholds for the extraction process appropriately.

\subsubsection{Target Visibility Constraints}

In this respect, the related constraints were categorized into two categories: those in relation to the image formation quality and the geometrical constraints. In this project, one of the objectives followed by the proposed algorithm was to find a workaround for the restrictions resulted from the point visibility constraint. Radiometric parameters of the camera were adjusted for maximum visual improvement of the images with the help of the camera's capability to identify the best configurations for the particular imaging conditions.

The target visibility-related geometrical constraints depend on three principal parameters: (1) field-of-view of the camera, (2) incidence angle constraint and (3) visibility goal. Given the object-camera distances encountered in this project, no too tight incidence angle was experienced. In addition, conducting the imaging stage during daytime, no practical problem occurred regarding the point visibility on the captured images. Performed via UAV photogrammetry imaging, no restriction was dealt in relation to the visibility goal.

\subsubsection{User Accessibility Constraints}

Given the use of UAV and openness of the project site, no special restriction in terms of user accessibility was reported. Moreover, the fact that the project workshop was shut down during the photogrammetric imaging, no such problem as the machinery-induced heat or limitations were encountered. 


\subsection{Flying the $U A V$ and Imaging}

In the present research, the required images were acquired with the help of a Phantom 3 Pro, a quad rotor, rotary wing UAV manufactured by DJI Company. Figure 11 demonstrates the used UAV and the drone pilot (author) during the course of imaging for the purpose of this project. Permissions and certificates were necessary before the UAV could be flown within an urban area.
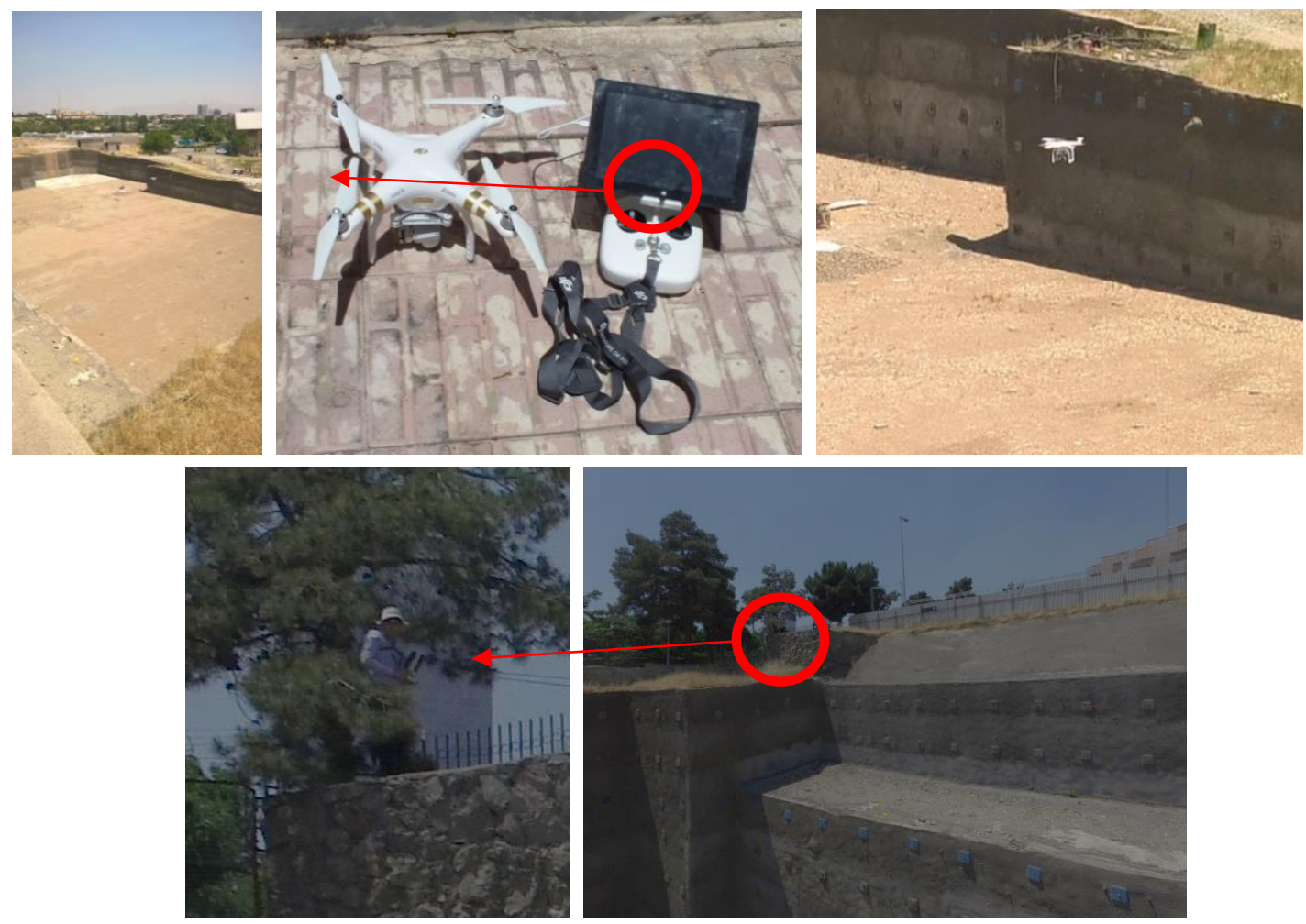

Figure 11. The Phantom 3 Pro when imaging the Seoul excavation pit, along with the drone pilot.

Installed on the drone was a Sony EXMOR $4 \mathrm{~K}$ camera (Figure 12) that could provide images of about 12 mega pixels in resolution. The camera type is" 12.4 Megapixel, 1/2.3-inch, EXMOR CMOS." The sensor size is " $6.16 \times 4.62 \mathrm{~mm}$," the focal length of the camera is " $\mathrm{f}=5 \mathrm{~mm}$ " and the pixel size of the camera images is" $4000 \times 3000$ (4:3)."

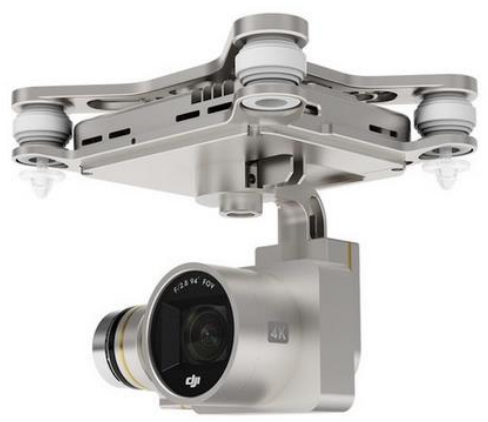

Figure 12. "Sony EXMOR 1/2.3"-4 K" camera mounted on phantom 3 professional.

The excavated wall was imaged according to the network design principles and calculations, as explained in the previous subsections, at three altitudes converged with respect to the feature. In each epoch, a total of about 20 images were captured. For instance, as observations, the images of the project captured by the Phantom 3 Pro are shown in Figure 13. 

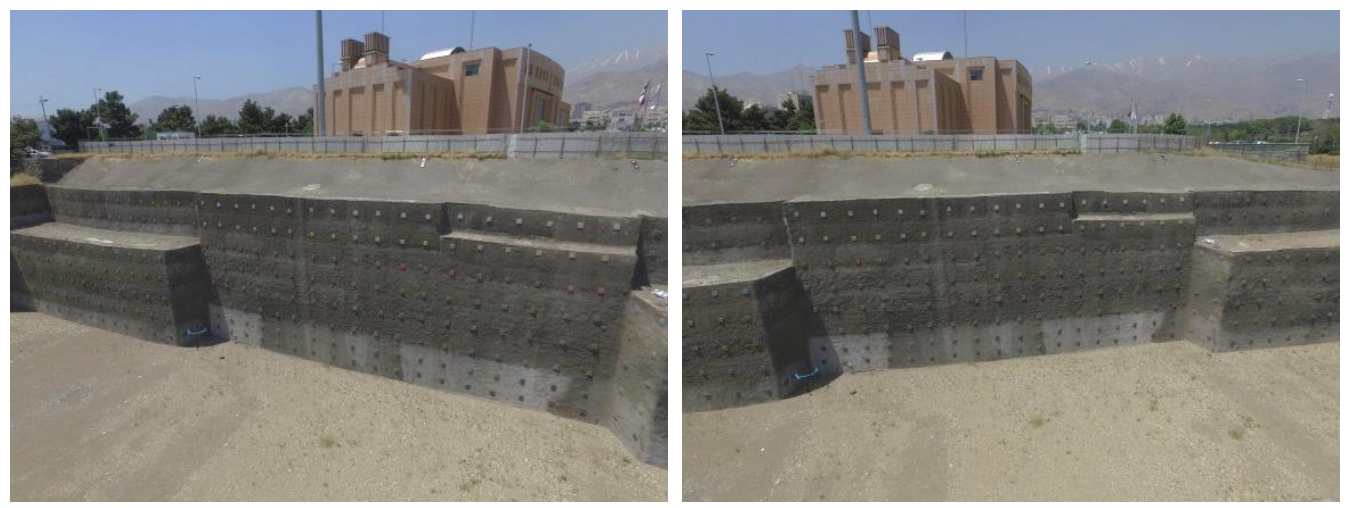

Figure 13. Sample observations captured using the Phantom 3 Pro for displacement monitoring of the excavated wall.

\subsection{Extraction of Image Points}

The proposed algorithm was applied to the observations captured in this project (according to the explanations given in Section 2.4). Once finished with extracting local features from the images using appropriate extraction parameters for the proposed algorithm, the extracted nails (Figure 14) were introduced into the next stage: points matching among the images.

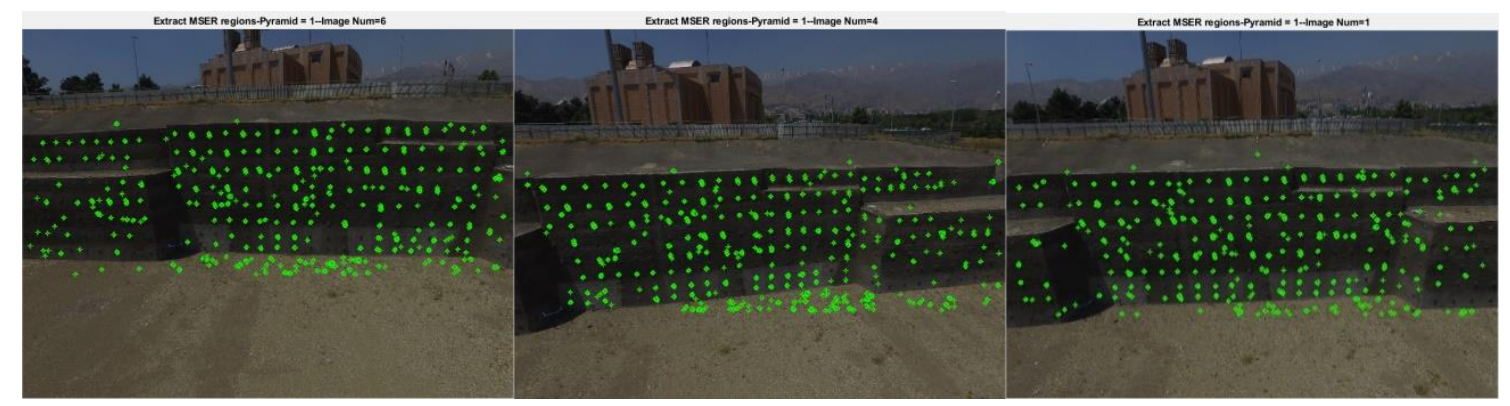

Figure 14. Sample results of local feature extraction from the UAV-captured images from the Seoul excavation project.

\subsection{Point Correspondence Matching}

The set of points extracted in the previous stage were introduced into the correspondence matching stage. According to the algorithm proposed in Section 2.4, a set of common points with high information content in all images were simultaneously matched. Figure 15 provides an example of correspondence matching via the proposed algorithm in Seoul excavation project.

As seen on Figure 15, the correspondence matching via the proposed algorithm returned acceptably precise results. That is, given the limited number of target points, the designation and precision of the matching among different images were visually inspected. Using the matched points on each epoch images, three-dimensional coordinates of the points and cameras positions calculated by bundle adjustment (Figure 16). The next step was to put the observations through the wall displacement monitoring process. 

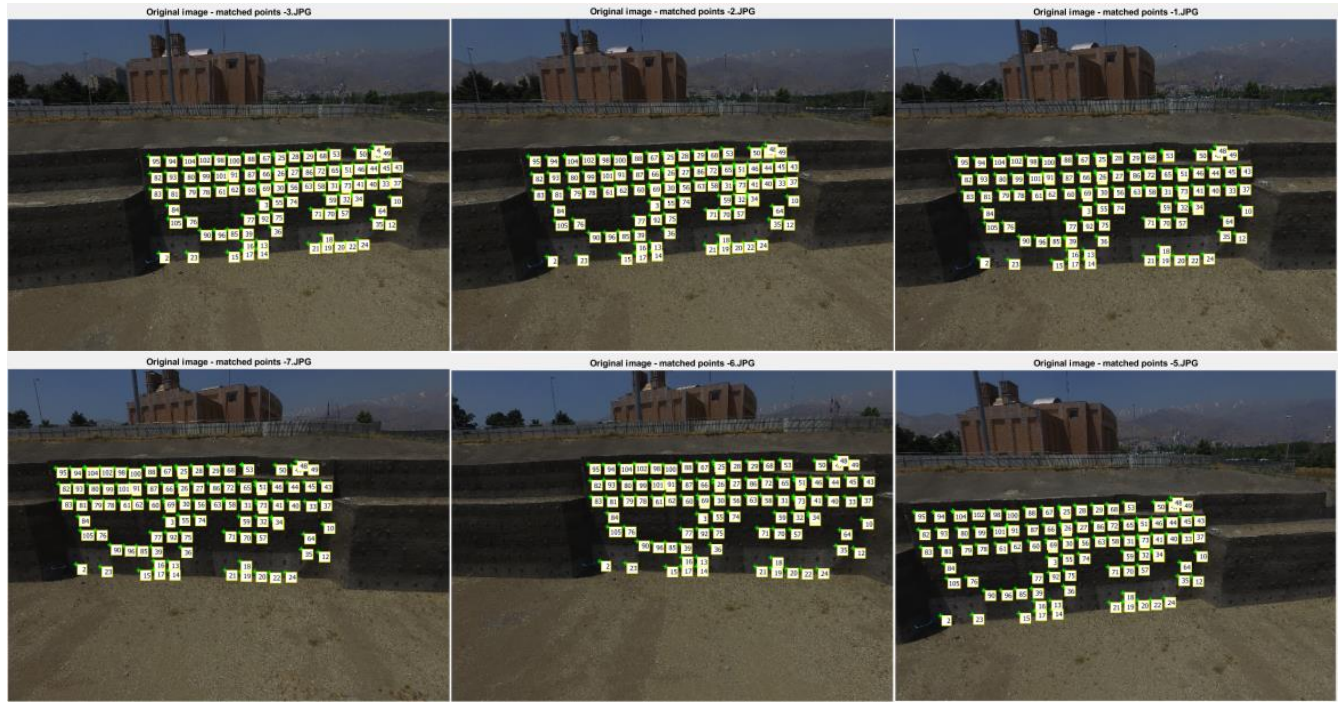

Figure 15. Example of the results of correspondence matching on the UAV-captured observations over the Seoul excavation project.

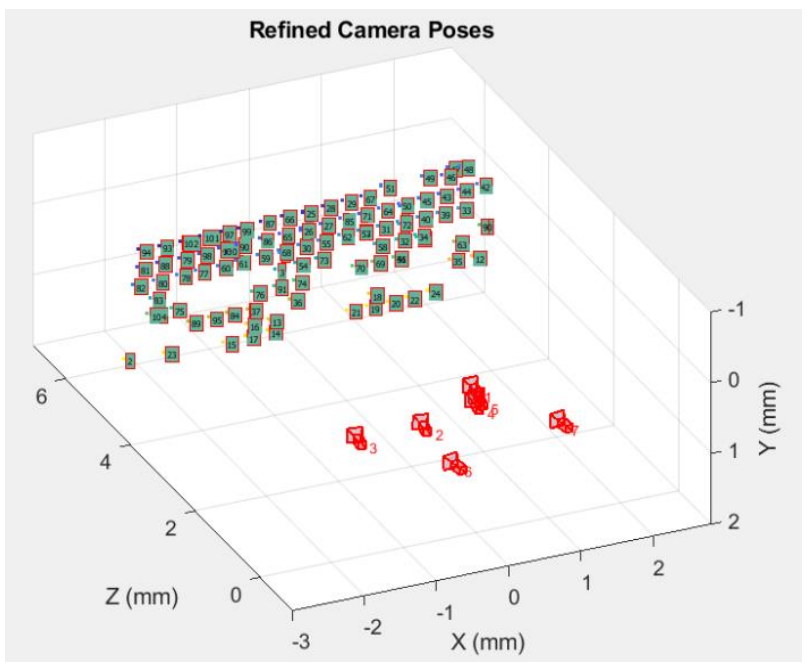

Figure 16. Generated three-dimensional coordinates of the points and some of the cameras (not in real scale).

\subsection{Displacement Measurement Using CPDA Method}

Once finished with performing the correspondence matching on the images of each epoch, the observations were ready for performing the displacement measurement process. According to the description of displacement measurement between two epochs in Section 2.5, the observations acquired in both epochs were simultaneously adjusted using the CPDA method. In the next step, 5 scale bars were used to control the network and check the results of adjustment in a one-by-one fashion, as demonstrated in Figure 17.

In this project, the two observation epochs were acquired successively with a time lag of about $10 \mathrm{~min}$. Given that the project was practically shut down during the time when the observation were getting acquired (and in fact, for the past several months), some zero displacement was expected between the two observation epochs. On this basis, the used methodology should be able to represent this zero displacement in its output. Once the image coordinates were extracted for each epoch and the set of observations were adjusted via CPDA, the estimated terrestrial coordinates for common points between the two epochs were expected to be identical, that is, with zero difference. Accordingly, average difference between the two epochs in coordinates of the on-feature points along the three 
dimensions would provide a measure of precision of the ultimate displacement measurement: the closer this value is to zero, the higher would be the accuracy of displacement measurement.
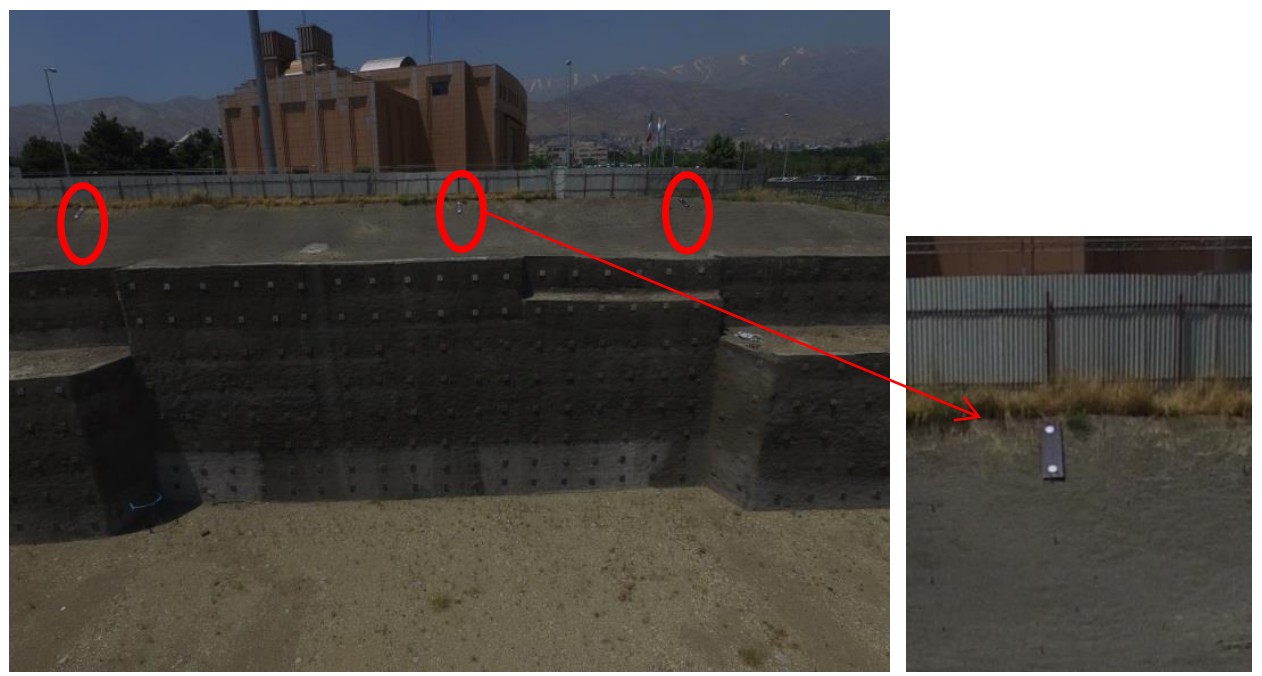

Figure 17. Examples of the scale bars used across the project area.

In displacement measurement, not only the validity of the measurement method is essential but also the precision of estimating terrestrial coordinates of points across the photogrammetric network adjusted via CPDA (both epochs together) along the three axes ( $x, y$ and $z$ ) is important. Table 2 presents the network robustness and point coordinate estimation precision in terms of mean standard deviation of the point coordinates estimation via CPDA in three dimensions.

Table 2. Precision of the combined network adjustment between the two epochs for displacement monitoring via CPDA methodology (in meters).

\begin{tabular}{|c|c|}
\hline $\begin{array}{l}\text { Method } \\
\text { Precision }\end{array}$ & CPDA Network Precision \\
\hline 0.007 & Precision $(X)$ \\
\hline 0.005 & Precision $(\mathrm{Y})$ \\
\hline 0.008 & Precision (Z) \\
\hline 0.0066 & Overall \\
\hline
\end{tabular}

According to Table 2, considering the adjustment via CPDA, average precision of estimating terrestrial coordinates of the point was about $7 \mathrm{~mm}$. The results of CPDA adjustment in terms of average difference in coordinates of the points on the wall between the two epochs are reported in Table 3. As mentioned, the closer the value of such differences to zero, the more accuracy would be the results of the displacement measurement.

Table 3. Average difference in measured wall displacement via photogrammetry between the two epochs (in meters).

\begin{tabular}{ccccc}
\hline $\begin{array}{c}\text { Prospect Displacement } \\
\text { on the Wall }\end{array}$ & $\begin{array}{c}\text { Mean of Displacement } \\
\text { on the Wall } \\
\text { (X Direction) }\end{array}$ & $\begin{array}{c}\text { Mean of Displacement } \\
\text { on the Wall } \\
\text { (Y Direction) }\end{array}$ & $\begin{array}{c}\text { Mean of Displacement } \\
\text { on the Wall } \\
\text { (Z Direction) }\end{array}$ & $\begin{array}{c}\text { Mean of Displacement } \\
\text { on the Wall }\end{array}$ \\
\hline 0 & 0.0104 & 0.0118 & 0.0113 & 0.0082 \\
\hline
\end{tabular}

According to Table 3, taking the approximately zero difference in wall displacement between the two epochs as a baseline, the displacement measured via photogrammetry turned out to suffer from an error of about $10 \mathrm{~mm}$. Naturally, part of this displacement measurement error is contributed from internal precision of the photogrammetric network (i.e., $7 \mathrm{~mm}$ ). 


\section{Discussion}

The results indicate that, for most part, the proposed method can provide the required accuracy for such projects with the help of local features on the wall. According to Equation (7) [19], maximum allowable wall displacement in Epoch 2, with an excavation wall height of $H=12 \mathrm{~m}$ and the ground covered dominantly with sandy soil $\left(\left(\delta_{h} / H\right)_{i}=1 / 500\right.$, as per Table 1$)$, was:

$$
\delta h=(\delta h / H) i \times H \rightarrow \delta h=\frac{1}{500} \times 12=0.024 \mathrm{~m}=2.4 \mathrm{~cm}
$$

Equation (7) implies that, in cases where wall displacements exceeding $\pm 2.4 \mathrm{~cm}$ occur between the two epochs, the project may be acknowledged as abnormal, requiring preventive actions such as construction of trusses, reducing the nail spacing, increasing the nail length or using other auxiliary methods such as cross bracing or anchorage to prevent the wall collapse. This $\pm 2.4 \mathrm{~cm}$ critical displacement could be easily detected using the proposed UAV photogrammetry displacement measurement algorithm. According to Table 3, we could determine the displacements with an accuracy of about $\pm 10 \mathrm{~mm}$. Therefore, the propose method could satisfy the anticipated precisions in displacement measurement for the considered project.

\section{Conclusions}

Monitoring the smallest changes in excavated walls is of paramount importance for preventing possible incidents, such as wall collapse. In the present research, a UAV photogrammetry method was proposed for excavated wall displacement monitoring. In the proposed methodology, the photogrammetry network was designed based on the specific conditions of the project environment. Scale bars were installed on the wall and local features were identified as targets for wall displacement. In the next step, convergent imaging was performed in two epochs by a camera-equipped UAV across the designed network. Next, regional local features were extracted and matched using the proposed algorithm. Then the point displacements between the two epochs, determined using CPAD algorithm. The proposed algorithm was unique in that it used existing features on the wall, rather than artificial photogrammetry targets, to perform correspondence matching and hence displace measurement. The use of UAV made it possible to use the three-dimensional space in front of the wall and acquire images across a robust network. Application of a drone served as a tool to cope with limited work space and on the ground imaging stations that restricted the implementation of terrestrial photogrammetry. The results indicated that, the proposed algorithm could determine three-dimensional coordinates of the points on the wall with a precision of about $\pm 7 \mathrm{~mm}$. It also could calculate wall displacements with an accuracy of about $\pm 10 \mathrm{~mm}$. Given the low cost of acquiring observations via and the required equipment for and also rapidness and flexibility of the proposed photogrammetry-based methodology, as compared to the micro-geodesy or instrumentation methods, it can serve as a practical solution for excavated wall displacement monitoring.

Author Contributions: Conceptualization, F.E., H.E., M.S. and F.K.; methodology, F.E., H.E., M.S.; software, F.E.; validation, F.E. and F.K.; formal analysis, F.E. and M.S.; investigation, F.E. and H.E.; resources, F.E. and H.E.; data curation, F.E. and F.K.; writing-original draft preparation, F.E. and H.E.; writing-review and editing, F.E. and H.E.; supervision, H.E., M.S. and F.K..

Funding: This research was funded by Iran National Science Foundation (INSF) and this research received no external funding for APC.

Acknowledgments: Authors would like to appreciate Iran National Science Foundation for supporting this research. Also, authors would like to thank Vahid Alimoradi for his great efforts in providing project location for data collection.

Conflicts of Interest: The authors declare no conflict of interest. 


\section{References}

1. Miller, P.E. Applications of 3D Measurement from Images; Whittles Publishing: Scotland, UK, 2009; Volume 24, ISBN 1870325699.

2. Luhmann, T.; Robson, S.; Kyle, S.; Harley, I. Close Range Photogrammetry-Principles, Techniques and Applications; Whittles Publishing: Scotland, UK, 2006; ISBN 9781849950572.

3. Esmaeili, F.; Varshosaz, M.; Ebadi, H. Displacement measurement of the soil nail walls by using close range photogrammetry and introduction of CPDA method. Measurement 2013, 46, 3449-3459. [CrossRef]

4. Li, L.; Zhang, X.; Chen, G.; Lytton, R. Measuring unsaturated soil deformations during triaxial testing using a photogrammetry-based method. Can. Geotech. J. 2015, 53, 472-489. [CrossRef]

5. Xiao, Z.; Liang, J.; Yu, D.; Liu, J. Rapid three-dimension optical deformation measurement for transmission tower with different loads. Opt. Lasers Eng. 2010, 48, 869-876. [CrossRef]

6. Jiang, R.; Jauregui, D.V. Development of a digital close-range photogrammetric bridge deflection measurement system. Measurement 2010, 43, 1431-1438. [CrossRef]

7. Jiang, R. Development of a Digital Photogrammetric System for Bridge Deflection Measurement; New Mexico State University: Las Cruces, New Mexico, 2005.

8. Feng, T.; Mi, H.; Scaioni, M.; Qiao, G.; Lu, P.; Wang, W.; Tong, X.; Li, R. Measurement of Surface Changes in a Scaled-Down Landslide Model Using High-Speed Stereo Image Sequences. Photogramm. Eng. Remote Sens. 2016, 82, 547-557. [CrossRef]

9. Ozbek, M.; Rixen, D.J.; Erne, O.; Sanow, G. Feasibility of monitoring large wind turbines using photogrammetry. Energy 2010, 35, 4802-4811. [CrossRef]

10. Scaioni, M.; Barazzetti, L.; Giussani, A.; Previtali, M.; Roncoroni, F.; Alba, M.I. Photogrammetric techniques for monitoring tunnel deformation. Earth Sci. Inform. 2014, 7, 83-95. [CrossRef]

11. Alba, M.; Barazzetti, L.; Giussani, A.; Roncoroni, F.; Scaioni, M. Development and Testing of a Method for Tunnel Monitoring Via Vision Metrology. Int. Arch. Photogramm. Remote Sens. Spat. Inf. Sci. 2010, 38, 17-22.

12. Barazzetti, L.; Scaioni, M. Development and implementation of image-based algorithms for measurement of deformations in material testing. Sensors 2010, 10, 7469-7495. [CrossRef]

13. Fraser, C.S.; Woods, A.; Brizzi, D. Hyper redundancy for accuracy enhancement in automated close range photogrammetry. Photogramm. Rec. 2005, 20, 205-217. [CrossRef]

14. Cerminaro, D.J. Implementation of Photogrammetry to Improve Proactive Assessment of Retaining Walls along Transportation Corridors; Michigan Technological University: Houghton, MI, USA, 2014.

15. Luo, X.; Tan, H.; Lin, G.; Lian, Y. Strain Field Measurements Using Digital Photogrammetry for Large Inflatable Structures. Exp. Tech. 2017, 41, 453-462. [CrossRef]

16. Tsvetkov, R.; Shardakov, I.; Shestakov, A.; Gusev, G.; Epin, V. Deformation monitoring of load-bearing reinforced concrete beams. Procedia Struct. Integr. 2017, 5, 620-626. [CrossRef]

17. Nocerino, E.; Menna, F.; Remondino, F.; Saleri, R. Accuracy and block deformation analysis in automatic UAV and terrestrial photogrammetry-Lesson learnt. ISPRS Ann. Photogramm. Remote Sens. Spat. Inf. Sci. 2013, II-5/W1, 203-208. [CrossRef]

18. Naumann, M.; Bill, R.; Niemeyer, F.; Nitschke, E. Deformation analysis of dikes using unmannaed aerial systems (UAS). In Proceedings of the South Baltic Conference on Dredged Materials in Dike Construction, Rostock, Germany, 10-12 April 2014; ISBN 9783860094099.

19. Lazarte, C.A.; Elias, V.; Espinoza, R.D.; Sabatini, P.J. Geotechnical Engineering Circular No. 7: Soil Nail Walls; Federal Highway Administration: Washington, DC, USA, 2003.

20. Atkinson, K.B. Close Range Photogrammetry and Machine Vision Whittles Publishing; Whittles Publishing: Scotland, UK, 1996; ISBN 187032546X.

21. Rieke-Zapp, D.; Tecklenburg, W.; Peipe, J.; Hastedt, H.; Haig, C. Evaluation of the geometric stability and the accuracy potential of digital cameras-Comparing mechanical stabilisation versus parameterisation. ISPRS J. Photogramm. Remote Sens. 2009, 64, 248-258. [CrossRef]

22. Fraser, C.S. Network Design Considerations for Non-Topographic Photogrammetry. Photogramm. Eng. Remote Sens. 1984, 50, 1115-1126.

23. Saadatseresht, M.; Fraser, C.S.; Samadzadegan, F.; Azizi, A. Visibility analysis in vision metrology network design. Photogramm. Rec. 2004, 19, 219-236. [CrossRef] 
24. Saadatseresht, M.; Varshosaz, M. Visibility prediction based on artificial neural networks used in automatic network design. Photogramm. Rec. 2007, 22, 336-355. [CrossRef]

25. Mason, S. Expert system-based design of close-range photogrammetric networks. ISPRS J. Photogramm. Remote Sens. 1995, 50, 13-24. [CrossRef]

26. Schmid, C.; Mohr, R. Local grayvalue invariants for image retrieval. IEEE Trans. Pattern Anal. Mach. Intell. 1997, 19, 530-535. [CrossRef]

27. Tuytelaars, T.; Mikolajczyk, K. Local Invariant Feature Detectors: A Survey. Found. Trends ${ }^{\circledR}$ Comput. Graph. Vis. 2008, 3, 177-280. [CrossRef]

28. Tuytelaars, T.; Van Gool, L. Matching widely separated views based on affine invariant regions. Int. J. Comput. Vis. 2004, 59, 61-85. [CrossRef]

29. Tuytelaars, T.; van Gool, L. Wide Baseline Stereo Matching based on Local, Affinely Invariant Regions. BMVC 2000, 412 .

30. Matas, J.; Chum, O.; Urban, M.; Pajdla, T. Robust wide-baseline stereo from maximally stable extremal regions. Image Vis. Comput. 2004, 22, 761-767.

31. Hartley, R.; Zisserman, A. Multiple View Geometry in Computer Vision, 2nd ed.; Cambridge University Press: New York, NY, USA, 2003; ISBN 0521540518, 9780521540513.

32. Mason, S. Expert System-Based Design of Photogrammetric Networks; ETH: Zürich, Switzerland, 1994; ISBN 3-906513-58-0.

(C) 2019 by the authors. Licensee MDPI, Basel, Switzerland. This article is an open access article distributed under the terms and conditions of the Creative Commons Attribution (CC BY) license (http:/ / creativecommons.org/licenses/by/4.0/). 